\title{
ON ESTIMATING THE VARIANCE OF THE OFFSPRING DISTRIBUTION IN A SIMPLE BRANCHING PROCESS
}

\author{
C. C. HEYDE, Australian National University
}

\begin{abstract}
A single realization $\left\{Z_{n}, 0 \leqq n \leqq N+1\right\}$ of a supercritical Galton-Watson process (so called) is considered and it is required to estimate the variance of the offspring distribution. A prospective estimator $N^{-1} \sum_{j=1}^{N}\left(Z_{j+1}-\hat{m} Z_{j}\right)^{2} Z_{j}^{-1}$ is proposed, where $\hat{m}=Z_{N+1} / Z_{N}$, and is shown to be strongly consistent on the non-extinction set. A centrai limit result and an iterated logarithm result are provided to give information on the rate of convergence of the estimator. It is also shown that the estimation results are robust in the sense that they continue to apply unchanged in the case where immigration occurs. Martingale limit theory is employed at each stage in obtaining the limit results.

BRANCHING PROCESSES; GALTON-WATSON PROCESSES; ESTIMATION; CONSISTENCY; CENTRAL LIMIT THEOREM; ITERATED LOGARITHM LAW; MARTINGALES; ROBUSTNESS
\end{abstract}

\section{Introduction}

Let $Z_{0}=1, Z_{1}, Z_{2}, \cdots$ denote a supercritical simple branching process of the kind that is commonly but inaccurately termed Galton-Watson process (see [8]). Suppose that $1<E Z_{1}=m$ and $0<\operatorname{var} Z_{1}=\sigma^{2}<\infty$. In this case it is well known that there exists a non-degenerate random variable $W$ such that $\lim _{n \rightarrow \infty}$ $m^{-n} Z_{n}=W$ almost surely (a.s.). It is also well known, and easily deducible from the above a.s. convergence result, how to estimate the mean $m$ of the offspring distribution on the basis of a single realization $\left\{Z_{n}, 0 \leqq n \leqq N+1\right\}$. In fact, $Z_{N+1} Z_{N}^{-1} \stackrel{\text { a.s. }}{\rightarrow} m$ on the non-extinction set $\{W>0\}$. Alternatively, the maximum likelihood estimator $\sum_{j=1}^{N+1} Z_{j} / \Sigma_{j=0}^{N} Z_{j}$ may be used and, again on the nonextinction set $\{W>0\}, \sum_{j=1}^{N+1} Z_{j} / \sum_{j=0}^{N} Z_{j} \stackrel{\text { a.s. }}{\rightarrow} m$ (see Heyde [4]). The maximum likelihood estimator, however, does not appear to have any special optimality properties in this context.

In this paper we shall be concerned with the estimation of $\sigma^{2}$, the variance of the offspring distribution, on the basis of the sample $\left\{Z_{n}, 0 \leqq n \leqq N+1\right\}$. This issue does not appear to have been discussed previously in the literature, save that Nagaev [10] observed that in the critical case, $m=1,\left(Z_{N+1}-Z_{N}\right)^{2} / Z_{N}$ could be used to estimate $\sigma^{2}$. We propose the estimator

$$
N^{-1} \sum_{j=1}^{N}\left(Z_{j+1}-\hat{m} Z_{j}\right)^{2} Z_{j}^{-1}
$$

Received 25 July 1973. 
where $\hat{m}=Z_{N+1} / Z_{N}$, show that it is strongly consistent for $\sigma^{2}$ on the nonextinction set $\{W>0\}$ and obtain a central limit result and an iterated logarithm result which give information on its rate of convergence to $\sigma^{2}$. We also show that the estimation procedure is robust in the sense that the results all continue to hold if an immigration component is introduced into the branching process.

\section{Results}

Theorem 1. Let $\hat{m}=Z_{N+1} Z_{N}^{-1}$. Then, on the non-extinction set $\{W>0\}$,

as $N \rightarrow \infty$.

$$
N^{-1} \sum_{j=1}^{N}\left(Z_{j+1}-\hat{m} Z_{j}\right)^{2} Z_{j}^{-1} \stackrel{\text { a.s. }}{\rightarrow} \sigma^{2}
$$

Proof. There is no essential loss of generality in giving the proof for the case $P\left(Z_{1}=0\right)=0$ in which case $Z_{n} \rightarrow \infty$ a.s. as $n \rightarrow \infty$. This we shall do for the sake of convenience. When $P\left(Z_{1}=0\right)>0$ it is well known that $Z_{n} \rightarrow \infty$ a.s. on the set $\{W>0\}$.

Let $\mathscr{F}_{n}$ be the $\sigma$-field generated by $Z_{1}, \cdots, Z_{n}$ and put

$$
U_{n+1}=\left(Z_{n+1}-m Z_{n}\right)^{2} Z_{n}^{-1}-\sigma^{2} \text {. }
$$

Then, since we can write

$$
Z_{n+1}=Z_{1}^{(1)}+Z_{1}^{(2)}+\cdots+Z_{1}^{\left(Z_{n}\right)}
$$

where the $Z_{1}^{(i)}$ are independent and identically distributed (i.i.d.) each with the distribution of $Z_{1}$, we have

$$
E\left(U_{n+1} \mid \mathscr{F}_{n}\right)=E\left\{Z_{n}^{-1}\left(\left(Z_{1}^{(1)}-m\right)+\cdots+\left(Z_{1}^{\left(Z_{n}\right)}-m\right)\right)^{2} \mid \mathscr{F}_{n}\right\}-\sigma^{2}=0
$$

and $E\left|U_{n+1}\right| \leqq 2 \sigma^{2}$ so that the $U_{n}$ are martingale differences. We prove (1) via a strong law of large numbers for martingales.

First note that, using (2) and (3),

$$
U_{n+1} \stackrel{\mathscr{g}}{=} Z_{n}^{-1} S_{Z_{n}}^{2}-\sigma^{2}
$$

where $S_{n}=\sum_{i=1}^{n} X_{i}, n \geqq 1$, say, the $X_{i}$ being i.i.d. each with the distribution of $Z_{1}-m$ (" $\mathscr{D}$ " denotes "in distribution"). Then, using Theorem 1 of Heyde and Leslie [6],

$$
\begin{aligned}
P\left(\left|U_{n+1}\right|>x\right) & =P\left(\left|Z_{n}^{-1} S_{Z_{n}}^{2}-\sigma^{2}\right|>x\right) \\
& \leqq\left\{\begin{array}{cl}
1, & 0 \leqq x \leqq \sigma^{2}, \\
P\left(S_{Z_{n}}^{2}>Z_{n} x\right), & x>\sigma^{2},
\end{array}\right. \\
& \leqq \begin{array}{cl}
1, & 0 \leqq x \leqq \sigma^{2}, \\
2\left[1-\Phi\left(x^{\frac{1}{2}} \sigma^{-1}\right)+d_{n}\right], & x>\sigma^{2},
\end{array}
\end{aligned}
$$


where $\left\{d_{n}\right\}$ is a certain sequence of positive constants with $\Sigma_{n} d_{n}<\infty$ and $\Phi(\cdot)$ is the distribution function of the unit normal law, $N(0,1)$.

Now we put $U_{n}^{\prime}=U_{n} I\left(\left|U_{n}\right| \leqq n\right), n \geqq 1$, and then, using (5),

$$
\begin{aligned}
\sum_{n=1}^{\infty} n^{-2} E\left(U_{n}^{\prime}-E\right. & \left.\left(U_{n}^{\prime} \mid \mathscr{F}_{n-1}\right)\right)^{2} \\
& \leqq \sum_{n=1}^{\infty} n^{-2} E\left(U_{n}^{\prime}\right)^{2} \\
& \leqq 2 \sum_{n=1}^{\infty} n^{-2} \int_{0<x \leqq n} x P\left(\left|U_{n}\right|>x\right) d x \\
& \leqq 2 \sum_{n=1}^{\left[\sigma^{2}\right]} n^{-2} \int_{0}^{n} x d x \\
& +2 \sum_{n=\left[\sigma^{2}\right]+1}^{\infty} n^{-2}\left\{\int_{0}^{\sigma^{2}} x d x+2 \int_{\sigma^{2}}^{n} x\left(1-\Phi\left(x^{\frac{1}{2}} \sigma^{-1}\right)+d_{n-1}\right) d x\right\} \\
& \leqq\left[\sigma^{2}\right]+4 \sigma^{4} \sum_{n=1}^{\infty} n^{-2}+2 \sum_{n=0}^{\infty} d_{n}<\infty
\end{aligned}
$$

after some algebra. Here $\left[\sigma^{2}\right]$ denotes the integer part of $\sigma^{2}$. Thus, using the standard Kolmogorov convergence criterion for martingales (e.g., Loève [9], p. 387),

$$
n^{-1} \sum_{k=1}^{n}\left\{U_{k}^{\prime}-E\left(U_{k}^{\prime} \mid \mathscr{F}_{k-1}\right)\right\} \stackrel{\text { a.s. }}{\rightarrow} 0
$$

as $n \rightarrow \infty$.

Next, again using (5) we have

$$
\sum_{k=1}^{\infty} P\left(U_{k} \neq U_{k}^{\prime}\right)=\sum_{k=1}^{\infty} P\left(\left|U_{k}\right|>k\right)<\infty
$$

provided that

$$
\sum_{k=1}^{\infty}\left\{1-\Phi\left(k^{\frac{1}{2}} \sigma^{-1}\right)\right\}<\infty
$$

which certainly holds since

$$
1-\Phi\left(k^{\frac{1}{2}} \sigma^{-1}\right) \sim(2 \pi)^{-\frac{1}{2}} \sigma k^{-\frac{1}{2}} e^{-\frac{1}{2} \sigma^{2} k}
$$

as $k \rightarrow \infty$. Thus, the sequences $\left\{U_{k}\right\}$ and $\left\{U_{k}^{\prime}\right\}$ are tail equivalent and hence from (6),

$$
n^{-1} \sum_{k=1}^{n}\left\{U_{k}-E\left(U_{k}^{\prime} \mid \mathscr{F}_{k-1}\right)\right\} \stackrel{\text { a.s. }}{\rightarrow} 0 .
$$


Now we shall show that

$$
E\left(U_{n}^{\prime} \mid \mathscr{F}_{n-1}\right) \stackrel{\text { a.s. }}{\rightarrow} 0
$$

as $n \rightarrow \infty$. Then of course

$$
n^{-1} \sum_{k=1}^{n} E\left(U_{k}^{\prime} \mid \mathscr{F}_{k-1}\right) \stackrel{\text { a.s. }}{\rightarrow} 0
$$

so that from (7),

$$
n^{-1} \sum_{k=1}^{n} U_{k} \stackrel{\text { a.s. }}{\rightarrow} 0
$$

which gives

$$
N^{-1} \sum_{k=1}^{N}\left(Z_{k+1}-m Z_{k}\right)^{2} Z_{k}^{-1} \stackrel{\text { a.s. }}{\rightarrow} \sigma^{2} .
$$

To prove (8) we note that, using the martingale property,

$$
\begin{aligned}
E\left(U_{n}^{\prime} \mid \mathscr{F}_{n-1}\right)= & -E\left(U_{n} I\left(\left|U_{n}\right|>n\right) \mid \mathscr{F}_{n-1}\right) \\
= & \sigma^{2} P\left(\left|U_{n}\right|>n \mid \mathscr{F}_{n-1}\right) \\
& -Z_{n-1}^{-1} E\left(\left(Z_{n}-m Z_{n-1}\right)^{2} I\left(\left|U_{n}\right|>n\right) \mid \mathscr{F}_{n-1}\right)
\end{aligned}
$$

and

$$
P\left(\left|U_{n}\right|>n \mid \mathscr{F}_{n-1}\right) \stackrel{\text { a.s. }}{\rightarrow} 0
$$

since

$$
\sum_{n=1}^{\infty} E P\left(\left|U_{n}\right|>n \mid \mathscr{F}_{n-1}\right)=\sum_{n=1}^{\infty} P\left(\left|U_{n}\right|>n\right)<\infty
$$

as shown above while, for $n>\sigma^{2}$,

$$
\begin{aligned}
& Z_{n-1}^{-1} E\left(\left(Z_{n}-m Z_{n-1}\right)^{2} I\left(\left|U_{n}\right|>n\right) \mid \mathscr{F}_{n-1}\right) \\
& \quad \leqq Z_{n-1}^{-1} E\left(\left(Z_{n}-m Z_{n-1}\right)^{2} I\left(\left(Z_{n}-m Z_{n-1}\right)^{2}>n Z_{n-1}\right) \mid \mathscr{F}_{n-1}\right) \\
& \quad \stackrel{\text { a.s. }}{\rightarrow} 0
\end{aligned}
$$

as $n \rightarrow \infty$, recalling (4) and since $\left\{S_{k}^{2} / k\right\}$ is uniformly integrable. The uniform integrability of $\left\{S_{k}^{2} / k\right\}$ is a simple consequence of Theorem 5.4 of Billingsley [1].

Finally, we have 


$$
\begin{aligned}
& N^{-1} \sum_{k=1}^{N}\left(Z_{k+1}-\hat{m} Z_{k}\right)^{2} Z_{k}^{-1} \\
& \quad=N^{-1} \sum_{k=1}^{N}\left(Z_{k+1}-m Z_{k}\right)^{2} Z_{k}^{-1}+2 N^{-1}(m-\hat{m}) \sum_{k=1}^{N}\left(Z_{k+1}-m Z_{k}\right) \\
& \quad+N^{-1}(m-\hat{m})^{2} \sum_{k=1}^{N} Z_{k} .
\end{aligned}
$$

The first term on the right-hand side of (10) tends a.s. to $\sigma^{2}$ by (9) so to complete the proof of (1) we need to show that the second and third terms on the right-hand side of (10) tend a.s. to zero.

To deal with the first of these, we note that

$$
E\left(Z_{j+1} \mid \mathscr{F}_{j}\right)=m Z_{j}
$$

so that $\left\{\sum_{j=1}^{N}\left(Z_{j+1}-m Z_{j}\right), \mathscr{F}_{N+1}\right\}$ is a martingale. Also,

$$
E\left(\left(Z_{j+1}-m Z_{j}\right)^{2} \mid \mathscr{F}_{j}\right)=Z_{j} \sigma^{2}
$$

so that

$$
E\left(Z_{j+1}-m Z_{j}\right)^{2}=\sigma^{2} m^{j}
$$

and

$$
\sum_{j=1}^{\infty} m^{-j} j^{-1} E\left(Z_{j+1}-m Z_{j}\right)^{2}<\infty
$$

which ensures, via the Kolmogorov condition for martingales,

$$
m^{-\frac{1}{2} N} N^{-\frac{3}{4}} \sum_{j=1}^{N}\left(Z_{j+1}-m Z_{j}\right) \stackrel{\text { a.s. }}{\rightarrow} 0 .
$$

Furthermore, Theorem 2 of [6] shows that

$$
\hat{m}-m=Z_{N+1} Z_{N}^{-1}-m=\sigma \zeta(N)\left(2 Z_{N}^{-1} \log N\right)^{\frac{1}{2}}
$$

where $\zeta(N)$ has its set of a.s. limit points confined to $[-1,1]$ with lim $\sup _{N \rightarrow \infty}$ $\zeta(N)=+1$ a.s., $\lim \inf _{N \rightarrow \infty} \zeta(N)=-1$ a.s. Then, using (11) and (12),

$$
\begin{aligned}
N^{-1}(\hat{m}-m) \sum_{k=1}^{N}\left(Z_{k+1}-m Z_{k}\right) \\
=\sigma\left\{\zeta(N)(2 \log N)^{\frac{1}{2}} N^{-\frac{1}{4}}\right\}\left\{\left(m^{N} Z_{N}^{-1}\right)^{\frac{1}{2}}\right\} \\
\quad \times\left\{m^{-\frac{1}{2} N} N^{-\frac{3}{4}} \sum_{j=1}^{N}\left(Z_{j+1}-m Z_{j}\right)\right\} \stackrel{\text { as. }}{\rightarrow} 0 .
\end{aligned}
$$

For the third term on the right-hand side of (10) we have, using (12) again, 


$$
\begin{aligned}
& N^{-1}(m-\hat{m})^{2} \sum_{j=1}^{N} Z_{j} \\
& \quad=2 \sigma^{2}\left\{\zeta^{2}(N) N^{-1} \log N\right\}\left\{m^{N} Z_{N}^{-1}\right\}\left\{m^{-N} \sum_{j=1}^{N} Z_{j}\right\} \stackrel{\text { a.s. }}{\rightarrow} 0
\end{aligned}
$$

since $m^{-N} \sum_{j=1}^{N} Z_{j} \stackrel{\text { a.s. }}{\rightarrow} m W /(m-1)$ by Theorem 3 of Heyde [4]. The proof of Theorem 1 is then completed by using (9), (13) and (14) in (10).

Next we proceed to a central limit result which provides a rate of convergence result for the estimator of Theorem 1.

Theorem 2. Suppose that $E Z_{1}^{4}<\infty$. Then, as $N \rightarrow \infty$,

$$
P\left(N^{\frac{1}{2}}\left(2 \sigma^{4}\right)^{-1}\left\{N^{-1} \sum_{j=1}^{N}\left(Z_{j+1}-\hat{m} Z_{j}\right)^{2} Z_{j}^{-1}-\sigma^{2}\right\} \leqq x \mid Z_{N}>0\right) \rightarrow \Phi(x) .
$$

Proof. Again we shall just give the proof for the case $P\left(Z_{1}=0\right)=0$. We first set out to prove

$$
N^{\frac{1}{2}}\left(2 \sigma^{4}\right)^{-1}\left\{N^{-1} \sum_{j=1}^{N}\left(Z_{j+1}-m Z_{j}\right)^{2} Z_{j}^{-1}-\sigma^{2}\right\} \stackrel{\mathscr{M}}{\rightarrow} N(0,1)
$$

and then show that we can replace $m$ by its estimator $\hat{m}$. The central limit result is obtained from a central limit theorem for martingales (Theorem 2 of Brown [3]) applied to the martingale $\left\{\sum_{j=1}^{N} U_{j}, \mathscr{F}_{N}\right\}$.

Using (3) and writing $T_{Z_{n}}=\left(Z_{1}^{(1)}-m\right)+\cdots+\left(Z_{1}^{\left(Z_{n}\right)}-m\right)$,

$$
\begin{aligned}
E\left(U_{n+1}^{2} \mid \mathscr{F}_{n}\right) & =E\left(\left(Z_{n}^{-1} T_{Z_{n}}^{2}-\sigma^{2}\right)^{2} \mid \mathscr{F}_{n}\right) \\
& =E\left(Z_{n}^{-2} T_{Z_{n}}^{4} \mid \mathscr{F}_{n}\right)-\sigma^{4}
\end{aligned}
$$

and

$$
\begin{aligned}
& E\left(Z_{n}^{-2} T_{Z_{n}}^{4} \mid \mathscr{F}_{n}\right) \\
& \quad=E\left\{Z_{n}^{-2}\left(\sum_{i=1}^{Z_{n}}\left(Z_{1}^{(i)}-m\right)^{4}+6 \sum_{Z_{n} \geqq i>j \geqq 1}\left(Z_{1}^{(i)}-m\right)^{2}\left(Z_{1}^{(j)}-m\right)^{2}\right) \mid \mathscr{F}_{n}\right\} \\
& \quad=Z_{n}^{-1} E\left(Z_{1}-m\right)^{4}+3 \sigma^{4}\left(1-Z_{n}^{-1}\right) \stackrel{\text { a.s. }}{\rightarrow} 3 \sigma^{4}
\end{aligned}
$$

as $n \rightarrow \infty$ so that

$$
n^{-1} \sum_{j=1}^{n} E\left(U_{j}^{2} \mid \mathscr{F}_{j-1}\right) \stackrel{\text { a.s. }}{\rightarrow} 2 \sigma^{4} .
$$

Also, since $E Z_{n}^{-1} \leqq \gamma^{n}$ for some $\gamma, 0<\gamma<1$ (see the proof of Theorem 2 of [5]),

$$
n^{-1} \sum_{j=1}^{n} E\left(U_{j}^{2}\right) \rightarrow 2 \sigma^{4}
$$


Thus, writing

$$
s_{n}^{2}=E\left(\sum_{j=1}^{n} U_{j}^{2}=\sum_{j=1}^{n} E U_{j}^{2}\right.
$$

we have

$$
s_{n}^{-2} \sum_{j=1}^{n} E\left(U_{j}^{2} \mid \mathscr{F}_{j-1}\right) \stackrel{\text { 2.s. }}{\rightarrow} 1
$$

as $n \rightarrow \infty$.

Next, we need to show that a Lindeberg condition is satisfied. In fact, we need

$$
s_{n}^{-2} \sum_{j=1}^{n} E\left(U_{j}^{2} I\left(\left|U_{j}\right| \geqq \varepsilon s_{n}\right)\right) \rightarrow 0
$$

as $n \rightarrow \infty$ for all $\varepsilon>0$ and this is clearly satisfied if

$$
s_{n}^{-2} \sum_{j=1}^{n} E\left(U_{j}^{2} I\left(\left|U_{j}\right| \geqq \varepsilon s_{j}\right)\right) \rightarrow 0
$$

and hence, in view of (16), if

$$
E\left(U_{n}^{2} I\left(\left|U_{n}\right| \geqq \eta n^{\frac{1}{2}}\right)\right) \rightarrow 0
$$

as $n \rightarrow \infty$ for all $\eta>0$.

Now, using (4) and for $n$ sufficiently large,

$$
\begin{aligned}
E\left(U_{n}^{2} I\left(\left|U_{n}\right| \geqq \eta n^{\frac{1}{2}}\right)\right) & \\
= & E\left(\left(Z_{n-1}^{-1} S_{Z_{n-1}}^{2}-\sigma^{2}\right)^{2} I\left(\left|Z_{n-1}^{-1} S_{Z_{n-1}}^{2}-\sigma^{2}\right|>\eta n^{\frac{1}{2}}\right)\right) \\
\leqq & 2 E\left(Z_{n-1}^{-2} S_{Z_{n-1}}^{4} I\left(Z_{n-1}^{-2} S_{Z_{n-1}}^{4}>\eta^{2} n\right)\right) \\
& +2 \sigma^{4} P\left(S_{Z_{n-1}}^{2}>\eta n^{\frac{1}{2}} Z_{n-1}\right) .
\end{aligned}
$$

The first term on the right-hand side of (19) tends to zero as $n \rightarrow \infty$ in view of the uniform integrability of $\left\{S_{k}^{4} / k^{2}\right\}$, which in turn follows, for example, from Theorem 5 of Brown [2] and Theorem 5.4 of Billingsley [1]. That the second term on the right-hand side of (19) tends to zero as $n \rightarrow \infty$ follows immediately from Theorem 1 of Heyde and Leslie [6].

The required conditions are thus satisfied ((17) and (18)) for the application of Theorem 2 of [3] which gives

that is,

$$
s_{N}^{-2} \sum_{j=1}^{N} U_{j} \stackrel{\mathscr{P}}{\rightarrow} N(0,1)
$$

$$
\left(2 \sigma^{4}\right)^{-1} N^{\frac{1}{2}}\left\{N^{-1} \sum_{j=1}^{N}\left(Z_{j+1}-m Z_{j}\right)^{2} Z_{j}^{-1}-\sigma^{2}\right\} \stackrel{\mathscr{M}}{\rightarrow} N(0,1) .
$$


Finally, we need to show that we can replace $m$ by $\hat{m}$ in (20). We have

$$
\begin{aligned}
\left(2 \sigma^{4}\right)^{-1} N^{\frac{1}{2}}\left\{N^{-1} \sum_{j=1}^{N}\left(Z_{j+1}-\hat{m} Z_{j}\right)^{2} Z_{j}^{-1}-\sigma^{2}\right\} \\
=\left(2 \sigma^{4}\right)^{-1} N^{\frac{1}{2}}\left\{N^{-1} \sum_{j=1}^{N}\left(Z_{j+1}-m Z_{j}\right)^{2} Z_{j}^{-1}-\sigma^{2}\right\} \\
+2\left(2 \sigma^{4}\right)^{-1} N^{-\frac{1}{2}}(m-\hat{m}) \sum_{j=1}^{N}\left(Z_{j+1}-m Z_{j}\right) \\
+\left(2 \sigma^{4}\right)^{-1} N^{-\frac{1}{2}}(m-\hat{m})^{2} \sum_{j=1}^{N} Z_{j}
\end{aligned}
$$

and in view of (20) it certainly suffices to show that

$$
N^{-\frac{1}{2}}(m-\hat{m}) \sum_{j=1}^{N}\left(Z_{j+1}-m Z_{j}\right) \stackrel{\text { a.s. }}{\rightarrow} 0
$$

and

$$
N^{-\frac{1}{2}}(m-\hat{m})^{2} \sum_{j=1}^{N} Z_{j} \stackrel{\text { a.s. }}{\rightarrow} 0 .
$$

The full force of these a.s. convergence results will be used later.

To prove (21) we first note that, in view of (12) and $Z_{N} / m^{N} \stackrel{\text { a.s. }}{\rightarrow} W$,

$$
m^{-\frac{1}{2} N}(\log N)^{-1}\left(Z_{N+1}-m Z_{N}\right) \stackrel{\text { a.s. }}{\rightarrow} 0 .
$$

Thus, using the Toeplitz Lemma (e.g., Loève [9], p. 238),

$$
\left(\sum_{j=1}^{N} m^{\frac{1}{2} j} \log j\right)^{-1} \sum_{j=1}^{N}\left(Z_{j+1}-m Z_{j}\right) \stackrel{\text { a.s. }}{\rightarrow} 0
$$

and

$$
\begin{aligned}
& N^{-\frac{1}{2}}(\hat{m}-m) \sum_{j=1}^{N}\left(Z_{j+1}-m Z_{j}\right) \\
& =\sigma\left\{\zeta(N) N^{-\frac{1}{2}}(2 \log N)^{\frac{1}{2}} m^{-\frac{1}{2} N}\left(\sum_{j=1}^{N} m^{\frac{1}{2} j} \log j\right)\right\} \\
& \quad \times\left\{\left(m^{N} Z_{N}^{-1}\right)^{\frac{1}{2}}\right\}\left\{\left(\sum_{j=1}^{N} m^{\frac{1}{2} j} \log j\right)^{-1} \sum_{j=1}^{N}\left(Z_{j+1}-m Z_{j}\right)\right\} \\
& \quad \stackrel{\text { a.s. }}{\rightarrow} 0
\end{aligned}
$$

since 


$$
\begin{aligned}
m^{-\frac{1}{2} N} N^{-\frac{1}{2}}(\log N)^{\frac{1}{2}} & \sum_{j=1}^{N} m^{\frac{1}{3} j} \log j \\
& \sim m^{-\frac{1}{2} N} N^{-\frac{1}{2}}(\log N)^{\frac{1}{2}} \int_{1}^{N} \exp \left\{x \log m^{\frac{1}{2}}\right\} \log x d x \\
& \sim\left(\log m^{\frac{1}{2}}\right)^{-1}(\log N)^{\frac{3}{2}} N^{-\frac{1}{2}} \rightarrow 0 .
\end{aligned}
$$

The result (22) is proved by noting that

$$
\begin{aligned}
N^{-\frac{1}{2}}(m-\hat{m})^{2} & \sum_{j=1}^{N} Z_{j} \\
& =2 \sigma^{2}\left\{\zeta^{2}(N) N^{-\frac{1}{2}} \log N\right\}\left\{m^{N} Z_{N}^{-1}\right\}\left\{m^{-N} \sum_{j=1}^{N} Z_{j}\right\} \stackrel{\text { a.s. }}{\rightarrow} 0
\end{aligned}
$$

as with (14). This completes the proof of the theorem.

Another type of rate of convergence result for the estimation procedure of Theorem 1 is provided by the following iterated logarithm result which gives, in a sense, a second term in the asymptotic expansion.

Theorem 3. Suppose that $E Z_{1}^{4+\delta}<\infty$ for some $\delta>0$. Then, on the nonextinction set $\{W>0\}$,

$$
N^{-1} \sum_{j=1}^{N}\left(Z_{j+1}-\hat{m} Z_{j}\right)^{2} Z_{j}^{-1}=\sigma^{2}+\eta(N)\left(4 \sigma^{4} N^{-1} \log \log N\right)^{\frac{1}{2}}
$$

where $\eta(N)$ has its set of a.s. limit points confined to $[-1,1]$ with lim $\sup _{N \rightarrow \infty}$ $\eta(N)=+1$ a.s., $\lim \inf _{N \rightarrow \infty} \eta(N)=-1$ a.s.

Proof. Again we shall just give the proof for the case $P\left(Z_{1}=0\right)=0$. The result is obtained with the aid of an iterated logarithm result for martingales (Theorem 1 of Heyde and Scott [7]) applied to the martingale $\left\{\sum_{j=1}^{N} U_{j}, \mathscr{F}_{N}\right\}$.

Again putting

$$
s_{n}^{2}=E\left(\sum_{j=1}^{n} U_{j}\right)^{2}=\sum_{j=1}^{n} E U_{j}^{2}
$$

what we need to check in order to apply Theorem 1 of [7] is that

$$
\begin{aligned}
& \sum_{n=1}^{\infty} s_{n}^{-4} E\left(U_{n}^{4} I\left(\left|U_{n}\right|<\delta s_{n}\right)\right)<\infty \text { for some } \delta>0, \\
& \sum_{n=1}^{\infty} s_{n}^{-1} E\left(\left|U_{n}\right| I\left(\left|U_{n}\right| \geqq \varepsilon s_{n}\right)\right)<\infty \text { for all } \varepsilon>0,
\end{aligned}
$$

and

$$
s_{n}^{-2} \sum_{k=1}^{n} U_{k}^{2} \stackrel{\text { a.s. }}{\rightarrow} 1 \quad \text { as } n \rightarrow \infty .
$$


That (23) holds is easily seen from (16) and a minor modification of the argument used to show that

$$
\sum_{n=1}^{\infty} n^{-2} E\left(U_{n}^{\prime}\right)^{2}<\infty
$$

in the proof of Theorem 1.

To show that (24) holds we firstly note that, using (4) and the $c_{r}$ - inequality (e.g., Loève [9], p. 155),

$$
\begin{aligned}
E\left|U_{n}\right|^{2+\frac{1}{2} \delta} & =E\left|Z_{n-1}^{-1} S_{Z_{n-1}}^{2}-\sigma^{2}\right|^{2+\frac{1}{2} \delta} \\
& \leqq 2^{1+\frac{1}{2} \delta}\left\{E\left(Z_{n-1}^{-\frac{1}{2}}\left|S_{Z_{n-1}}\right|\right)^{4+\delta}+\sigma^{4+\delta}\right\} \leqq C<\infty
\end{aligned}
$$

for some $C>0$ where we have employed Theorem 5 of [2] to uniformly bound $E\left(Z_{n-1}^{-\frac{1}{2}}\left|S_{Z_{n-1}}\right|\right)^{4+\delta}$. Then, using Markov's inequality,

$$
P\left(\left|U_{n}\right| \geqq x\right) \leqq x^{-2-\frac{1}{2} \delta} E\left|U_{n}\right|^{2+\frac{1}{2} \delta} \leqq C x^{-2-\frac{1}{2} \delta} .
$$

In view of (16), the result (24) holds since, for any $\eta>0$,

$$
\begin{aligned}
& \sum_{n=1}^{\infty} n^{-\frac{1}{2}} E\left(\left|U_{n}\right| I\left(\left|U_{n}\right| \geqq \eta n^{\frac{1}{2}}\right)\right) \\
& \quad \leqq \eta \sum_{n=1}^{\infty} P\left(\left|U_{n}\right| \geqq \eta n^{\frac{1}{2}}\right)+\sum_{n=1}^{\infty} n^{-\frac{1}{2}} \int_{\eta \sqrt{ } n}^{\infty} P\left(\left|U_{n}\right|>x\right) d x \\
& \quad \leqq \eta C \sum_{n=1}^{\infty}\left(\eta n^{\frac{1}{2}}\right)^{-2-\frac{1}{2} \delta}+C \sum_{n=1}^{\infty} n^{-\frac{1}{2}} \int_{\eta \sqrt{ } n}^{\infty} x^{-2-\frac{1}{2} \delta} d x<\infty .
\end{aligned}
$$

To prove (25) we need to show that

$$
n^{-1} \sum_{j=1}^{n}\left\{U_{j}^{2}-E\left(U_{j}^{2} \mid \mathscr{F}_{j-1}\right)\right\} \stackrel{\text { a.s. }}{\rightarrow} 0
$$

and then the required result follows using (16) and (17). The result (27) is obtained by paralleling much of the argument used to derive the strong law for martingales in the proof of Theorem 1 . In fact, now defining $U_{n}^{\prime}=U_{n}^{2} I\left(U_{n}^{2} \leqq n\right), n \geqq 1$, we can mimic the previous argument up to the point of obtaining

$$
n^{-1} \sum_{k=1}^{n}\left\{U_{k}^{2}-E\left(U_{k}^{\prime} \mid \mathscr{F}_{k-1}\right)\right\} \stackrel{\text { a.s. }}{\rightarrow} 0 .
$$

The only extra piece of information we need in order to achieve this is that $\sum_{n=1}^{\infty} n^{2} d_{n}<\infty$ and this holds since $d_{n}$ decreases geometrically under the present moment conditions ([5], Theorem 2). Next we have, using (26), 


$$
\begin{array}{rl}
\sum_{n=1}^{\infty} n^{-1} & E\left(E\left(U_{n}^{2} I\left(U_{n}^{2}>n\right) \mid \mathscr{F}_{n-1}\right)\right) \\
& =\sum_{n=1}^{\infty} n^{-1} E\left(U_{n}^{2} I\left(U_{n}^{2}>n\right)\right) \\
& \leqq \sum_{n=1}^{\infty} P\left(U_{n}^{2}>n\right)+\sum_{n=1}^{\infty} n^{-1} \int_{n}^{\infty} P\left(U_{n}^{2}>x\right) d x \\
& \leqq C \sum_{n=1}^{\infty} n^{-1-\frac{1}{4} \delta}+C \sum_{n=1}^{\infty} n^{-1} \int_{n}^{\infty} x^{-1-\frac{1}{4} \delta} d x<\infty .
\end{array}
$$

Thus, using the Kronecker lemma together with (29),

$$
n^{-1} \sum_{k=1}^{n} E\left(U_{k}^{2} I\left(U_{k}^{2}>k\right) \mid \mathscr{F}_{k-1}\right) \stackrel{\text { a.s. }}{\rightarrow} 0
$$

and hence (27) follows via (28).

Theorem 1 of [7] then gives

$$
\begin{aligned}
& \limsup _{N \rightarrow \infty} \frac{\sum_{j=1}^{N}\left(Z_{j+1}-m Z_{j}\right)^{2} Z_{j}^{-1}-N \sigma^{2}}{\left(2 s_{N}^{2} \log \log s_{N}^{2}\right)^{\frac{1}{2}}}=1 \text { a.s., } \\
& \underset{N \rightarrow \infty}{\lim \inf _{j=1}} \frac{\sum_{j+1}^{N}\left(Z_{j+1}-m Z_{j}\right)^{2} Z_{j}^{-1}-N \sigma^{2}}{\left(2 s_{N}^{2} \log \log s_{N}^{2}\right)^{\frac{1}{2}}}=-1 \text { a.s., }
\end{aligned}
$$

and hence, using (16), (21) and (22),

$$
\begin{aligned}
& \limsup _{N \rightarrow \infty} \frac{\sum_{j=1}^{N}\left(Z_{j+1}-\hat{m} Z_{j}\right)^{2} Z_{j}^{-1}-N \sigma^{2}}{\left(4 \sigma^{4} N \log \log N\right)^{\frac{1}{2}}}=1 \text { a.s., } \\
& \underset{N \rightarrow \infty}{\lim \inf _{j=1}} \frac{\sum_{j=1}^{N}\left(Z_{j+1}-\hat{m} Z_{j}\right)^{2} Z_{j}^{-1}-N \sigma^{2}}{\left(4 \sigma^{4} N \log \log N\right)^{\frac{1}{2}}}=-1 \text { a.s. }
\end{aligned}
$$

The required result then follows.

\section{The process with immigration}

The properties of the estimator

$$
\hat{\sigma}^{2}(\hat{m})=N^{-1} \sum_{j=1}^{N}\left(Z_{j+1}-\hat{m} Z_{j}\right)^{2} Z_{j}^{-1},
$$

where $\hat{m}=Z_{N+1} Z_{N}^{-1}$, are robust to the extent that $\hat{\sigma}^{2}(\hat{m})$ continues to estimate the variance of the offspring distribution and the results of Theorems 2 and 3 continue to hold even if the branching process is one with immigration. Only minor modifications of the proofs of Theorems 1,2 and 3 are necessary to establish these results. 
Again we define

$$
U_{n+1}=\left(Z_{n+1}-m Z_{n}\right)^{2} Z_{n}^{-1}-\sigma^{2} .
$$

In the case with immigration we can write

$$
Z_{n+1}=Z_{1}^{(1)}+\cdots+Z_{1}^{\left(Z_{n}\right)}+I_{n+1}
$$

where there $Z_{1}^{(i)}$ are i.i.d. each with the offspring distribution and are independent of $Z_{n}$ while the $I$ 's are i.i.d. each with the immigration distribution, $I_{n+1}$ being independent of $Z_{n}$. The sequence $\left\{U_{n}, \mathscr{F}_{n}\right\}$ is no longer a sequence of martingale differences since

$$
\begin{aligned}
E\left(U_{n+1} \mid \mathscr{F}_{n}\right) & =E\left\{Z_{n}^{-1}\left(\left(Z_{1}^{(1)}-m\right)+\cdots+\left(Z_{1}^{\left(Z_{n}\right)}-m\right)+I_{n+1}\right)^{2} \mid \mathscr{F}_{n}\right\}-\sigma^{2} \\
& =Z_{n}^{-1} E I^{2}
\end{aligned}
$$

but we can of course form a sequence of martingale differences by taking

$$
\left\{U_{n}-E\left(U_{n} \mid \mathscr{F}_{n-1}\right), \mathscr{F}_{n}\right\} \text {. }
$$

The required results are obtained by working with the martingale

$$
\left\{\sum_{j=1}^{n}\left[U_{j}-E\left(U_{j} \mid \mathscr{F}_{j-1}\right)\right], \mathscr{F}_{n}\right\}
$$

and then showing that the conditional expectations can be discarded. For example, to obtain the strong consistency result $\hat{\sigma}^{2}(\hat{m}) \stackrel{\text { a.s }}{\rightarrow} \sigma^{2}$ it is a matter of showing

$$
N^{-1} \sum_{j=1}^{N}\left\{U_{j}-E\left(U_{j} \mid \mathscr{F}_{j-1}\right)\right\} \stackrel{\text { a.s. }}{\rightarrow} 0
$$

while

$$
N^{-1} \sum_{j=1}^{N} E\left(U_{j} \mid \mathscr{F}_{j-1}\right) \stackrel{\text { a.s. }}{\rightarrow} 0
$$

and

$$
\hat{\sigma}^{2}(m)-\hat{\sigma}^{2}(\hat{m}) \stackrel{\text { a.s. }}{\rightarrow} 0 .
$$

The proof of (30) parallels the proof of the corresponding result in the proof of Theorem 1. Theorem 3 of [6] is used instead of Theorem 1 of [6]. The proof of (31) follows simply from the Kronecker lemma since $\sum_{j=1}^{\infty} j^{-1} Z_{j}^{-1}<\infty$ a.s. as a consequence of $\sum_{j=1}^{\infty} j^{-1} E Z_{j}^{-1}<\infty$. The proof of (32) again follows that of the corresponding result in the proof of Theorem 1. Theorem 4 of [6] is used to represent the difference $\hat{m}-m$ in this case. Similar considerations also apply for the central limit and iterated logarithm results but the details do not bear repetition and are omitted.

It is worth remarking that the estimator $\hat{m}$ also possesses robustness properties of the type outlined above. The paper [6] provides the results which lead to this conclusion. 


\section{References}

[1] Billingsley, P. (1968) Convergence of Probability Measures. Wiley, New York.

[2] Brown, B. M. (1970) Characteristic functions, moments and the central limit theorem. Ann. Math. Statist. 41, 658-664.

[3] Brown, B. M. (1971) Martingale central limit theorems. Ann. Math. Statist. 42, 59-66.

[4] Heyde, C. C. (1970) Extension of a result of Seneta for the super-critical Galton-Watson process. Ann. Math. Statist. 41, 739-742.

[5] Heyde, C. C. ANd Brown, B. M. (1971) An invariance principle and some convergence rate results for branching processes. $Z$. Wahrscheinlichkeitsth. 20, 271-278.

[6] Heyde, C. C. AND Leslie, J. R. (1971) Improved classical limit analogues for GaltonWatson processes with or without immigration. Bull. Austral. Math. Soc. 5, 145-155.

[7] Heyde, C. C. ANd Scott, D. J. (1973) Invariance principles for the law of the iterated logarithm for martingales and processes with stationary increments. Ann. Prob. 1, 428-436.

[8] Heyde, C. C. And Seneta, E. (1972) The simple branching process, a turning point test and a fundamental inequality: A historical note on I. J. Bienaymé. Biometrika, 59, 680-683.

[9] Loغ̀ve, M. (1963) Probability Theory. (3rd ed.) Van Nostrand, Princeton, N. J.

[10] NaGaEv, A. V. (1967) On estimating the number of direct descendants of a particle in a branching process. Theor. Probability Appl. 12, 314-320. 